\title{
Transformación, fragmentación y continuidad del sistema de partidos de Castilla-La Mancha (2003-2015)
}

Transformation, fragmentation and continuity of the party system of Castilla-La Mancha (2003-2015)

\author{
José María Ramírez Dueñas \\ Universidad Complutense de Madrid. Madrid/España \\ https://orcid.org/0000-0002-4760-4034 \\ josemara@ucm.es
}

Recibido/Received: 16/05/2020

Modificado/Modified: 15/11/2020

Aceptado/Accepted: 18/12/2020

\section{RESUMEN}

La literatura académica española ha manifestado poco interés por el sistema de partidos de Castilla-La Mancha, al contrario que los del resto de Comunidades Autónomas, especialmente de aquellas consideradas históricas. En el presente texto se pretende analizar la evolución del comportamiento electoral de los ciudadanos de la región para observar la transformación del sistema de partidos desde la hegemonía electoral del Partido Socialista (2003 y 2007), la llegada de la alternancia política con la entrada del Partido Popular en la Junta (2011) y la vuelta al poder del PSOE (2015). En base a las encuestas poselectorales del Centro de Investigaciones Sociológicas (CIS) de las elecciones autonómicas, se han trazado algunas de las explicaciones para entender los alineamientos partidistas de la región. Los resultados indican que el éxito del PSOE está relacionado con una mayor abstención diferencial de los votantes del PP, así como por su voto dual en las elecciones autonómicas debido a una buena valoración del candidato socialista. Este modelo se ha ido debilitando hasta que en 2011 la gestión de la crisis económica acaba con el PSOE en la oposición. Además, la menor fragmentación electoral en la región en las elecciones de 2015 parece estar influido, más que por cuestiones ideológicas, por el rechazo de los liderazgos de los dos candidatos de los partidos sistémicos.

\section{PALABRAS CLAVE}

Castilla-La Mancha, elecciones, sistema de partidos, voto, comportamiento electoral, socialdemocracia, polarización política, PSOE, PP

\section{SUMARIO}

1. Introducción 2. El sistema de partidos en Castilla-La Mancha. 3.Metodología de análisis. 4. Resultados. 4.1. La hegemonía electoral del partido socialista (2003-2011). 4.2. El fin de la hegemonía: el primer Gobierno de cambio (2011-2015). 4.3. ¿La institucionalización del pluralismo moderado (2015-2019)? 5. Conclusiones. 6. Bibliografía.

\begin{abstract}
The party system in Castilla-La Mancha has been a little studied issue in Spanish academic literature, unlike the rest of the Autonomous Communities, especially those historical considered. In this article, we aim to analyze the evolution of the electoral behavior of the citizens of the region to observe the party system transformation, from the electoral hegemony of the PSOE (2003 and 2007) until the arrival of political alternation (2011) and the return to the socialists' power (2015). Based on the post-electoral surveys of the Center for Sociological Research (CIS) of the regional elections, we have outlined some
\end{abstract}


of the explanations to understand the partisan alignments of the region. The results indicate that the success of the PSOE is related to a greater differential abstention of the voters of the PP, as well as a dual vote of these by the PSOE in the regional elections, thanks in part to a good assessment among these voters of the socialist candidate. This model weakened until 2011, the management of the economic crisis and the devaluation of management will end the PSOE in the opposition. Furthermore, the model allows us to verify that the lower electoral fragmentation in the region after the 2015 elections, more than for ideological reasons, is related to the rejection by the leaders of the two candidates of the systemic parties.

\section{KEYWORDS}

Castilla-La Mancha, elections, party system, vote, electoral behavior, Social Democratic parties, political polarization, PSOE, PP.

\section{CONTENTS}

1. Introduction 2. The party system in Castilla-La Mancha. 3. Methodology of analysis. 4. Results. 4.1. The electoral hegemony of the Socialist Party (2003-2011). 4.2. The end of hegemony: the first government of change (2011-2015). 4.3. The institutionalization of moderate pluralism (2015-2019)? 5 . Conclusions. 6. References.

\section{INTRODUCCIÓN}

La investigación española acerca de los sistemas de partidos y las convocatorias electorales de nuestro Estado Autonómico ha sido amplia y detallada, especialmente la de las llamadas comunidades históricas. Los estudios sobre Cataluña (Montero y Font, 1991; Riba, 2000; Balcells I Ventura, 2007; Rivera y Jaráiz, 2016); País Vasco (Llera, 2006; Llera, Leonisio y García Ramadán, 2008a; o Llera, 2016); Galicia (Lago, 2000; Álvarez González, 2003; Groba y Pereira, 2018); Andalucía (Montabes, 1996; Montabes y Ortega, 2008; Ortega, García Hipola y Trujillo, 2013; Cazorla, 2014); Canarias (Corujo, Fernández-Esquer y Rama, 2019), etc. han puesto el foco en las dinámicas propias que hacen de sus sistemas de partidos y sus convocatorias electorales susceptibles de análisis sociológico. En cambio, ese foco no se ha detenido de igual manera en Castilla-La Mancha, pese a ser en sí mismo un escenario muy interesante para la investigación social, al tratarse de una de las comunidades donde la estabilidad política y partidista ha funcionado durante más de 25 años.

Existen varias razones para llevar a cabo un análisis detenido del sistema de partidos en la región. En primer lugar, por la estabilidad manifiesta que ha demostrado, pese a la sucesión de acontecimientos políticos, económicos y sociales que han tenido lugar en los últimos veinte años en Castilla-La Mancha como en el resto de España. Su sistema de partidos (que posteriormente detallaremos) se ha mantenido casi de forma inalterable, incluso tras la derrota electoral del hegemónico PSOE en las elecciones de las autonomías del régimen común de mayo de 2011 o la fragmentación del escenario nacional en 2015 con la aparición de los dos partidos emergentes, Podemos y Ciudadanos (Mena, 1998; Llera et al., 2008b; Mezo, 2019).

En segundo lugar, esta estabilidad se fundamenta en la hegemonía electoral en la región de los dos principales partidos a escala nacional (Partido Socialista y Partido Popular). Hasta las elecciones de 2011, incluidas éstas, ambos sumaban más del $90 \%$ de los votos en las elecciones autonómicas, alcanzando un máximo histórico de más del 95\% en las de 1999. La llegada de los nuevos partidos en 2015 y su consolidación en 2019 ha afectado en menor medida a este sistema de partidos que a los de otras autonomías: los dos partidos sistémicos siguen 
representando más del $72 \%$ de los votos en estas dos últimas convocatorias regionales, pese a tener menor porcentaje a nivel nacional en las pasadas elecciones al Congreso de los Diputados: 50-55\% (2015-2016) y 45\% (2019). La existencia de un posible voto dual hacia estos dos partidos en las autonómicas parece más que evidente: estas diferencias se acortan si comparamos ese $72 \%$ de votos obtenido por ambos en los comicios regionales de 2015 , frente al 66\% que sumaron PP y PSOE en las elecciones generales de 2015 o el 65\% en las de 2016. Algunas de las explicaciones podrían recaer en el sistema electoral aplicado en la región, sobre el cual, posteriormente, aportaremos más referencias. Sin desmerecer posibles explicaciones sobre sus efectos, este estudio pretende observar si existen otros criterios de naturaleza sociológica que puedan explicar la estabilidad partidista comentada con anterioridad.

Gráfico 1: Evolución del porcentaje de voto en las elecciones a las Cortes de Castilla-La Mancha (2003-2019)

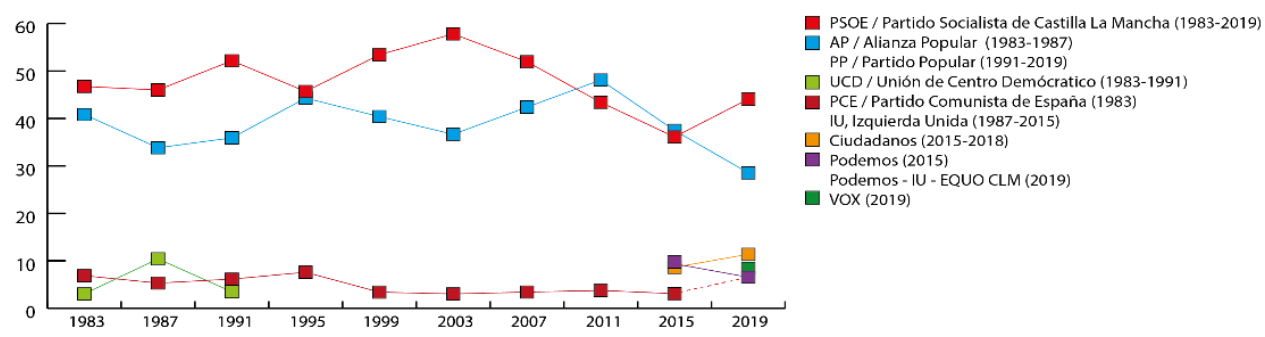

Elaboración propia. Fuente: Cortes de Castilla-La Mancha.

Como consecuencia de esta estabilidad, el sistema de partidos de la región no se ha visto afectado por la entrada de partidos de corte regionalista (como ha podido suceder en Cantabria, Andalucía, Comunidad Valenciana o Navarra, con diferentes posiciones ideológicas) o provinciales, pese a la complejidad que supuso la formación de la Autonomía. Si bien en ciertas autonomías esas tensiones históricas se han transformado en partidos provinciales (como Unión del Pueblo Leonés, en Castilla y León, que reclama la separación de la provincia del resto del ente autonómico), no se ha producido consecuencia electoral alguna en ese sentido en la región pese a las negativas iniciales de Guadalajara respecto a su inclusión en CastillaLa Mancha, si en ella no se integraba Madrid. Mientras que para Mena (1998), la ausencia de los PANES (Partidos de Ámbito No Estatal) en la Cámara castellano-manchega se debe a su nula penetración y escaso conocimiento social, Castellanos (2007) aporta como posible explicación la escasa intensidad de una identidad regional o identidades provinciales de tipo excluyente, incluso en aquellas provincias menos proclives a la formación de la autonomía.

Por último, el interés de estudio del comportamiento electoral en esta región se debe a los ciclos electorales propios de la región, que han funcionado de manera diferente a como se desarrollaban a nivel nacional: pese a la derrota electoral del PSOE en el año 2000, los mejores datos a nivel autonómico de este partido fueron en 1999 y 2003; o la recuperación del Gobierno regional en el año 2015, pese a que este partido a nivel nacional entraba en pleno retroceso (Mezo, 2019).

Este estudio tiene como objetivo analizar los movimientos electorales, así como las explicaciones del voto de los ciudadanos a nivel individual de la región en el conjunto de comicios durante el período de estudio, más concretamente, desde las elecciones autonómicas 
de 2003 hasta 2015. ¿Cuáles son las explicaciones a la hegemonía electoral del PSOE de Castilla-La Mancha durante siete convocatorias electorales consecutivas con mayoría absoluta? ¿Por qué el Partido Popular fue incapaz de convertirse en la alternativa de gobierno a los socialistas hasta las elecciones de 2011? ¿Por qué el gobierno conservador de María Dolores de Cospedal (2011-2015) no revalidó su gobierno en 2015? ¿Por qué su sistema de partidos, a pesar la llegada de Podemos y Ciudadanos, no se fragmentó de igual manera que en el resto de Comunidades?

Para dar respuesta a estas preguntas, en este texto se propone una primera aproximación al sistema de partidos de Castilla-La Mancha para ofrecer unas primeras hipótesis de trabajo para el modelo de estudio. Tras ello, mediante un modelo de análisis cuantitativo basado en las encuestas poselectorales del Centro de Investigaciones Sociológicas, se pretende a través de regresiones logísticas testar y observar los patrones de comportamiento que han guiado el comportamiento electoral de los castellano-manchegos a lo largo de estas dos décadas de análisis.

\section{EL SISTEMA DE PARTIDOS EN CASTILLA-LA MANCHA}

El sistema de partidos en Castilla-La Mancha, en términos de Sartori (1976), podría ser catalogado en, al menos, tres fases diferentes. En primer lugar, una primera etapa (1983-2011) de partido predominante en el que el PSOE regional domina tanto el poder legislativo (mayorías absolutas ininterrumpidas) como el poder ejecutivo (Junta de Comunidades) gracias a victorias electorales por más de 10 puntos porcentuales (21 puntos de diferencia en su máximo de 2003). La existencia de terceros partidos como Centro Democrático y Social (19871991) o Izquierda Unida (1991-1999) no supuso un impedimento para esta hegemonía electoral debido a que, en la práctica, no había alternancias posibles a este partido. Sólo en las elecciones de 1995, donde el PP arrasó en las elecciones municipales y conquistó varias comunidades (como Madrid, La Rioja o Murcia), la diferencia porcentual entre los dos principales partidos se redujo a algo más de un punto y los socialistas pudieron perder esta mayoría absoluta, que mantuvieron por escaso margen.

Tabla 1: Índices electorales y parlamentarios del análisis del sistema de partidos de CastillaLa Mancha

\begin{tabular}{|c|c|c|c|c|c|}
\hline & $\mathbf{2 0 0 3}$ & $\mathbf{2 0 0 7}$ & $\mathbf{2 0 1 1}$ & $\mathbf{2 0 1 5}$ & $\mathbf{2 0 1 9}$ \\
\hline $\begin{array}{c}\text { Número efectivo de Partidos Electorales (Laakso y } \\
\text { Taagapera) }\end{array}$ & 2,04 & 2,13 & 2,27 & 3,45 & 3,33 \\
\hline $\begin{array}{c}\text { Número efectivo de partidos parlamentarios (Laakso y } \\
\text { Taagapera) }\end{array}$ & 1,96 & 1,89 & 1,96 & 2,22 & 2,27 \\
\hline Volatilidad parlamentaria & 6,38 & 6,38 & 6,33 & 6,05 & 24,24 \\
\hline Volatilidad electoral agregada & 4,91 & 6,39 & 9,2 & 20,63 & 16,56 \\
\hline Fragmentación electoral (Rae) & 0,51 & 0,53 & 0,56 & 0,71 & 0,70 \\
\hline Fragmentación parlamentaria (Rae) & 0,49 & 0,47 & 0,49 & 0,55 & 0,56 \\
\hline
\end{tabular}

Elaboración Propia, en base a los criterios de Ruiz y Otero (2013).

La segunda etapa, y siguiendo el mismo modelo de clasificación de Sartori, podría ser considerada como de sistema bipartidista, una vez que se produce la ruptura de la hegemonía 
socialista y el cambio de partido en las instituciones, con un gobierno del Partido Popular (2011-2015). Bajo esta perspectiva, la competición entre las dos grandes formaciones se desarrolla en torno al eje ideológico para la obtención de una mayoría suficiente para un gobierno monocolor.

Por último, la última etapa se produce con la vuelta del PSOE castellano-manchego al poder de la mano de un acuerdo de investidura del candidato socialista, Emiliano García Page, con Podemos (2015-2019). Este modelo, denominado pluralismo moderado, la formación morada interviene en la constitución del Gobierno de la Junta, bien como apoyo externo (2015-2017), bien como parte del propio Gobierno (2017-2019), constituyéndose el primer gobierno de coalición en la región. Las elecciones de mayo de 2019, las cuales escapan a este estudio, constituyen una vuelta a la lógica bipartidista previa (2011-2015), aunque carecemos de herramientas suficientes para observar si los efectos serán permanentes o la competición electoral volverá a fases anteriores debido a la estabilización de terceros partidos.

Tabla 2: Resultados (porcentaje de voto y representación parlamentaria) del conjunto de partidos para el periodo de estudio a las elecciones autonómicas (1999-2019)

\begin{tabular}{|c|c|c|c|c|c|c|c|c|c|c|c|c|}
\hline & \multicolumn{2}{|c|}{1999} & \multicolumn{2}{|c|}{2003} & \multicolumn{2}{|c|}{2007} & \multicolumn{2}{|c|}{2011} & \multicolumn{2}{|c|}{2015} & \multicolumn{2}{|c|}{2019} \\
\hline & $\%$ & Esc & $\%$ & Esc & $\%$ & Esc & $\%$ & Esc & $\%$ & Esc & $\%$ & Esc \\
\hline PSOE & $\begin{array}{c}54,19 \\
\%\end{array}$ & 26 & $\begin{array}{c}58,61 \\
\%\end{array}$ & 29 & $\begin{array}{c}52,63 \\
\%\end{array}$ & 26 & $\begin{array}{c}44,14 \\
\%\end{array}$ & 24 & $\begin{array}{c}36,11 \\
\%\end{array}$ & 15 & $\begin{array}{c}44,10 \\
\%\end{array}$ & 19 \\
\hline $\mathbf{P P}$ & $\begin{array}{c}40,98 \\
\%\end{array}$ & 21 & $\begin{array}{c}37,16 \\
\%\end{array}$ & 18 & $\begin{array}{c}42.93 \\
\%\end{array}$ & 21 & $\begin{array}{c}48,92 \\
\%\end{array}$ & 25 & $\begin{array}{c}37,49 \\
\%\end{array}$ & 16 & $\begin{array}{c}28,53 \\
\%\end{array}$ & 10 \\
\hline Izquierda Unida & $3,46 \%$ & 0 & $3,09 \%$ & 0 & $3,47 \%$ & 0 & $3,84 \%$ & 0 & $3,10 \%$ & 0 & $\mathrm{n} / \mathrm{a}$ & $\mathrm{n} / \mathrm{a}$ \\
\hline Ciudadanos & & & & & & & & & $8,46 \%$ & 0 & $\begin{array}{c}11,38 \\
\%\end{array}$ & 4 \\
\hline $\begin{array}{l}\text { Podemos / Unidos } \\
\text { Podemos }\end{array}$ & & & & & & & & & $9,75 \%$ & 2 & $6,92 \%$ & 0 \\
\hline VOX & & & & & & & & & $0,48 \%$ & 0 & $7,02 \%$ & 0 \\
\hline Escaños totales & & 47 & & 47 & & 47 & & 49 & & 33 & & 33 \\
\hline Participación & $74,9 \%$ & & $76,2 \%$ & & $\begin{array}{c}73,70 \\
\%\end{array}$ & & $\begin{array}{c}75,96 \\
\%\end{array}$ & & $\begin{array}{c}71,50 \\
\%\end{array}$ & & $\begin{array}{c}67,98 \\
\%\end{array}$ & \\
\hline
\end{tabular}

Fuente: Cortes de Castilla-La Mancha.

El sistema electoral, ya mencionado con anterioridad, es clave para entender la configuración del sistema de partidos en la región y la consecución de mayorías absolutas, inclusive tras la reforma de 2014. La ley 5/1986 reflejaba el modelo: Cortes regionales formadas entre 40 y 47 diputados, circunscripción electoral fijada en la provincia, con un mínimo de 3 diputados y asignación del resto por población. La reforma del Estatuto de 1997 amplía el máximo de diputados regionales a 59 (ya previsto en el Estatuto de Autonomía de la región), y fija el mínimo de escaños de cada provincia: Ciudad Real (11), Toledo (11); Albacete (10); Cuenca (8) y Guadalajara (7); si bien la reforma de la ley electoral de 1998 mantuvo el número de escaños en la misma cifra (47), aumentados a 49 en la reforma de la ley electoral de 2007. Como ya se ha mencionado, el sistema carecía de proporcionalidad, sobrerrepresentando a las provincias menos pobladas, Cuenca y Guadalajara, y subrepresentando a las más pobladas, Toledo y Ciudad Real (Martín Sánchez, 2010).

Esta desproporcionalidad se incrementó en la reforma del Estatuto de Autonomía de 2014 que reduce el número de diputados en las Cortes de Castilla-La Mancha a 33, siendo esta región 
junto con La Rioja, la que menos representantes tienen en su parlamento regional. Nótese que el umbral efectivo para obtener representación es cercano al $8 \%$ de los votos en la circunscripción donde es más sencillo obtener el primer escaño, pese a que su normativa fija el umbral mínimo para obtener representación en el 3\% (Fernández Esquer, 2016).

Con este escenario de competición, se han trazado cuatro hipótesis de trabajo para explicar los cuatro comicios en línea con algunas investigaciones:

Hipótesis 1: El abstencionismo de los votantes del Partido Popular en las Elecciones Generales será mayor que entre aquellos que votan al PSOE, especialmente en las dos primeras elecciones donde este partido gana con mayoría absoluta (2003-2007).

Las elecciones regionales, consideradas históricamente como de segundo orden, han generado numerosos estudios acerca de la posible abstención diferencial de unos votantes por encima de otros. Por ejemplo, en el estudio de Riba (2000) se observó una menor participación en las elecciones autonómicas de los votantes de izquierda que votan al PSC; Trujillo y Ortega (2015) también detallan en Andalucía una alta correlación entre la participación electoral con el porcentaje de apoyo del PSOE-A, estando estos votantes más movilizados que los del Partido Popular de la región. En ese sentido, y si bien en Castilla-La Mancha la participación en las elecciones regionales ha sido tradicionalmente alta (la brecha entre ambas convocatorias electorales no es superior al 5\%, siendo una de las Comunidades donde menor es la diferencia de abstención), es probable la existencia de una posible abstención diferencial, esto es, que la alta estabilidad en el comportamiento de los ciudadanos y de la hegemonía electoral del PSOE de Castilla-La Mancha esté motivada por una mayor movilización por parte de sus votantes frente al resto de partidos, y más en concreto, los del Partido Popular, siendo además este último partido el ganador en todas las convocatorias electorales nacionales (Congreso de los Diputados) dentro del período de estudio (2000-2016) hasta las elecciones de abril y noviembre de 2019. 
Tabla 3: Resultados (porcentaje de voto y representación parlamentaria) del conjunto de partidos en las Elecciones Generales (datos para Castilla-La Mancha )

\begin{tabular}{|c|c|c|c|c|c|c|c|c|c|c|c|c|c|c|c|c|}
\hline & \multicolumn{2}{|c|}{2000} & \multicolumn{2}{|c|}{2004} & \multicolumn{2}{|c|}{2008} & \multicolumn{2}{|c|}{2011} & \multicolumn{2}{|c|}{2015} & \multicolumn{2}{|c|}{2016} & \multicolumn{2}{|c|}{$\begin{array}{l}2019 \\
(\mathrm{Ab}) \\
\end{array}$} & \multicolumn{2}{|c|}{$\begin{array}{l}2019 \\
(\mathrm{Nv}) \\
\end{array}$} \\
\hline & $\%$ & $\mathrm{E}$ & $\%$ & E & $\%$ & E & $\%$ & E & $\%$ & E & $\%$ & E & $\%$ & $\mathrm{E}$ & $\%$ & E \\
\hline PSOE & $\begin{array}{c}40,78 \\
\%\end{array}$ & 8 & $\begin{array}{c}46,5 \\
\%\end{array}$ & 9 & $\begin{array}{c}44,51 \\
\%\end{array}$ & 9 & $\begin{array}{c}30,34 \\
\%\end{array}$ & $\begin{array}{l}1 \\
4\end{array}$ & $\begin{array}{c}28,36 \\
\%\end{array}$ & 7 & $\begin{array}{c}27,28 \\
\%\end{array}$ & 7 & $\begin{array}{c}32,38 \\
\%\end{array}$ & 9 & $\begin{array}{c}33,1 \\
\%\end{array}$ & 9 \\
\hline $\mathbf{P P}$ & $\begin{array}{c}52,36 \\
\%\end{array}$ & $\begin{array}{l}1 \\
2\end{array}$ & $\begin{array}{c}47,4 \\
\%\end{array}$ & $\begin{array}{l}1 \\
1\end{array}$ & $\begin{array}{c}49,36 \\
\%\end{array}$ & $\begin{array}{l}1 \\
2\end{array}$ & $\begin{array}{c}55,81 \\
\%\end{array}$ & 7 & $\begin{array}{c}38,14 \\
\%\end{array}$ & $\begin{array}{l}1 \\
0\end{array}$ & $\begin{array}{c}42,73 \\
\%\end{array}$ & $\begin{array}{l}1 \\
2\end{array}$ & $\begin{array}{c}22,66 \\
\%\end{array}$ & 6 & $\begin{array}{c}26,87 \\
\%\end{array}$ & 7 \\
\hline $\begin{array}{c}\text { Izquierda } \\
\text { Unida }\end{array}$ & $\begin{array}{c}4,35 \\
\%\end{array}$ & 0 & $\begin{array}{c}3,37 \\
\%\end{array}$ & 0 & $\begin{array}{c}2,93 \\
\%\end{array}$ & 0 & $\begin{array}{c}5,78 \\
\%\end{array}$ & 0 & $\begin{array}{c}3,59 \\
\%\end{array}$ & 0 & & & & & & \\
\hline $\begin{array}{c}\text { Ciudadan } \\
\text { os }\end{array}$ & & & & & & & & & $\begin{array}{c}13,77 \\
\%\end{array}$ & 3 & $\begin{array}{c}13,04 \\
\%\end{array}$ & 0 & $\begin{array}{c}17,48 \\
\%\end{array}$ & 4 & $\begin{array}{c}6,84 \\
\%\end{array}$ & 0 \\
\hline $\begin{array}{l}\text { Podemos } \\
\text { / Unidos } \\
\text { Podemos }\end{array}$ & & & & & & & & & $\begin{array}{c}13,65 \\
\%\end{array}$ & 1 & $\begin{array}{c}14,74 \\
\%\end{array}$ & 2 & $\begin{array}{c}10,17 \\
\%\end{array}$ & 0 & $\begin{array}{c}9,22 \\
\%\end{array}$ & 0 \\
\hline VOX & & & & & & & & & & & & & $\begin{array}{c}15,28 \\
\%\end{array}$ & 2 & $\begin{array}{c}21,9 \\
\%\end{array}$ & 5 \\
\hline $\begin{array}{c}\text { Participa } \\
\text { ción }\end{array}$ & $\begin{array}{c}76,3 \\
\%\end{array}$ & & $\begin{array}{c}79,9 \\
\%\end{array}$ & & $80 \%$ & & $\begin{array}{c}75,8 \\
\%\end{array}$ & & $\begin{array}{c}75,3 \\
\%\end{array}$ & & $\begin{array}{c}71,8 \\
\%\end{array}$ & & $\begin{array}{c}78,02 \\
\%\end{array}$ & & $\begin{array}{c}71,36 \\
\%\end{array}$ & \\
\hline
\end{tabular}

Fuente: Ministerio del Interior. $\%=$ Porcentaje de voto a nivel autonómicos. E = Escaños conseguidos en el Congreso de los Diputados.

Hipótesis 2: El apoyo de los pequeños municipios (entornos rurales) al PSOE permite explicar buena parte de su éxito electoral hasta 2007.

En el análisis sobre la estabilidad partidista en aquellas Comunidades Autónomas donde el PSOE ha estado en el poder durante las últimas décadas hasta las elecciones de 2011 (Aragón, Extremadura, Asturias...), las posiciones tienden a relacionar su éxito electoral en sus mayores apoyos de los entornos rurales y pequeñas ciudades por este partido (frente a los grandes núcleos de población). En ese sentido, la línea de investigación dominante en Andalucía, principal foco de investigación por la fortaleza de este partido en la región, ha sido el mayor alineamiento y movilización electoral de las zonas rurales y entornos menos poblados con ciudadanos posicionados más a la izquierda en el eje, mientras que los votantes de las grandes ciudades se mantenían afines al Partido Popular en las elecciones autonómicas (Porras, 1984; Trujillo, Ortega y Montabes, 2015; Montabes, 2015). Castilla-La Mancha, compartiendo proximidad territorial y cultural, podría compartir este alineamiento ideológico. En ese sentido, será probable un mayor voto hacia el PSOE en las zonas rurales que en las grandes urbes.

Hipótesis 3: Los apoyos electorales del PSOE estarán relacionados con una buena valoración de su candidato. Además, consideramos que esta variable será explicativa para una parte de votantes del PP (en las Elecciones Generales) para apoyar al PSOE en las Autonómicas.

Los análisis han corroborado una estrecha relación entre simpatía a partido, la valoración de candidatos y el voto (Mata y Ortega, 2013; Rico, 2002, 2009). En ese sentido, y en línea con la "presidencialización" de la política a nivel nacional y la mayor impronta de los líderes como movilizadores en las campañas electorales, el fuerte protagonismo de José Bono 
(presidente de la Junta hasta 2004) podría conllevar que su valoración fuera una de las variables más explicativas para el voto hacia el PSOE en sus votantes; y no sólo en ellos, sino que también parece probable que su buena evaluación pudiera conllevar, por parte de aquellos que votan al PP en las Generales, un apoyo al PSOE en las Autonómicas.

Hipótesis 3.1.: Los mayores apoyos al Partido Popular y Partido Socialista (frente a Podemos y Ciudadanos) en 2015 están relacionados con los liderazgos de sus candidatos.

En línea con la hipótesis anterior, nuestra hipótesis para explicar la menor fragmentación del sistema de partidos de la región, así como de una mayor concentración de voto en los partidos sistémicos frente a los emergentes, está relacionada con los liderazgos. En ese sentido, la probabilidad de voto hacia estos partidos se reducirá con buenas valoraciones de los dos líderes.

\section{METODOLOGÍA DE ANÁLISIS}

El estudio del comportamiento electoral ha sido ampliamente desarrollado por la literatura académica, especialmente desde la II Guerra Mundial. Los enfoques aproximativos a la teoría del voto han sido fundamentalmente tres: el sociológico (también llamado modelo de Columbia, donde los ciudadanos expresan su posición social a través de su voto), el psicológico (escuela de Michigan, donde se reafirma el peso de las actitudes políticas por encima de las variables sociológicas más clásicas: identidad partidista, simpatía hacia candidatos, temas de campaña) y el económico (modelo de Rochester, donde los votantes apoyarán al candidato que más beneficios desde un punto de vista racional le reporte). El presente artículo presenta un modelo incorporando algunas de las variables que estas tres escuelas históricas han presentado como explicativas del voto.

Por ello, y con el fin de evaluar las tres hipótesis planteadas, hemos realizado un análisis cuantitativo-estadístico a través de una serie de variables para poder realizar su verificación. En ese sentido, y bajo el marco teórico propuesto, y en base a las encuestas poselectorales de las Elecciones Autonómicas de las cuatro convocatorias, hemos desarrollado un modelo de investigación mediante regresiones logísticas binarias con el voto a los diferentes partidos para comprobar las variables más explicativas entorno al voto de los castellano-manchegos durante el período de estudio. Debemos además tener en cuenta que, en primer lugar, las muestras de estas encuestas son más pequeñas que las que realiza el CIS a nivel nacional, por lo que, especialmente en las elecciones de 2015, el grupo de los votantes de los partidos emergentes es estadísticamente pequeño para poder establecer conclusiones significativas y sus datos han de tomarse aún con más cautela. En segundo lugar, las preguntas del cuestionario varían a lo largo de la década de estudio (2003-2015) por lo que, a pesar de guardar cierta cohesión y linealidad con los modelos de plan de análisis, en algunos casos han tenido que realizarse adaptaciones para poder incluir el conjunto de variables.

La variable dependiente del modelo de análisis es el recuerdo de voto de las elecciones autonómicas tomadas de manera dicotómica (1-Votó a este partido, 0-No lo votó) para todos los partidos con representación parlamentaria durante el periodo de análisis: Partido Socialista (PSOE), Partido Popular (PP) y Podemos (sólo en 2015), a lo que hemos sumado a Ciudadanos 
(2015) para poder observar la menor fragmentación electoral en esos comicios; así como la participación electoral (1-Participación, 0-Abstención).

Sobre estas dependientes hemos aplicado una serie de variables independientes y de control clásicas en el estudio del comportamiento electoral. En primer lugar, de carácter sociodemográfico: edad, sexo (1- hombre / 0-mujer), estudios, y una variable clave para contrastar la segunda hipótesis referida al factor contextual del voto en entornos rurales: el tamaño del municipio, ampliamente utilizada en los análisis de voto en Andalucía para obtener la correlación con el grado de urbanización.

En segundo lugar, se han incorporado en el modelo variables político-ideológicas para observar la relevancia del contexto electoral y la situación económica. Algunas son ampliamente utilizadas en análisis sociológico como, por ejemplo, el interés de la información política en campaña electoral, la evaluación de la situación económica, o la valoración retrospectiva del Gobierno autonómico, la cual no puede ser aplicada en el modelo de 2003 debido a la falta de la pregunta en el cuestionario del CIS. Además, el modelo de análisis incluye el recuerdo de voto de la convocatoria anterior en las Elecciones Autonómicas y Generales (Congreso de los Diputados) y la ubicación ideológica.

Por último, se han agregado diversas variables de valoración de líderes, más en concreto la evaluación que hace cada encuestado de los líderes de los dos principales partidos: el incumbent regional y el líder de la oposición (Partido Socialista y Partido Popular). Junto a ellos, se ha tomado la valoración del Presidente del Gobierno, cuando ha sido posible, en los modelos de análisis de las elecciones de 2007 y 2011; además del candidato de Podemos en los comicios de 2015, incorporado sólo en la regresión para sus propios votantes.

\section{RESULTADOS}

Como se apuntaba en el segundo apartado en el cual se analizaba el modelo de sistema de partidos instaurado en Castilla-La Mancha, se consideraron tres etapas diferenciadas: en primer lugar, un sistema de partido predominante (hasta el año 2011), bipartidista (2011-2015) y pluralista moderado (2015-2019).

\subsection{LA HEGEMONÍA ELECTORAL DEL PARTIDO SOCIALISTA (2003-2011)}

La primera fase de análisis, considerada como sistema de partido predominante, se centra en las dos últimas convocatorias electorales en las cuales el PSOE de Castilla-La Mancha obtiene mayorías absolutas en los comicios regionales. Durante la misma, debe considerarse la existencia de un posible voto dual, es decir, mientras que el ganador en el año 2003 fue el PSOE con un 54\%, las Elecciones Generales del año siguiente el Partido Popular venció en la región por algo más del 47\%. Este patrón se repitió en los comicios regionales de 2007 y en las generales de 2008. Los motivos de este deslizamiento de fuerzas están relacionados, primero, con la transversalidad ideológica que el PSOE despliega, que permite ensanchar sus bases electorales a zonas propias del Partido Popular; así como con las valoraciones positivas de los Presidentes de la Junta de Comunidades y candidatos por este partido, José Bono (2003) y José María Barreda (2007), por parte de los votantes del PP a las Elecciones Generales. Ambos hechos confirmarían el voto dual de una parte de los electores conservadores hasta el año 2007 (voto al PP en las Elecciones Generales, voto al PSOE en las Autonómicas). 
Gráfico 2: Probabilidad de abstenerse, votar PSOE o PP a las Elecciones de Castilla-La Mancha (2003)
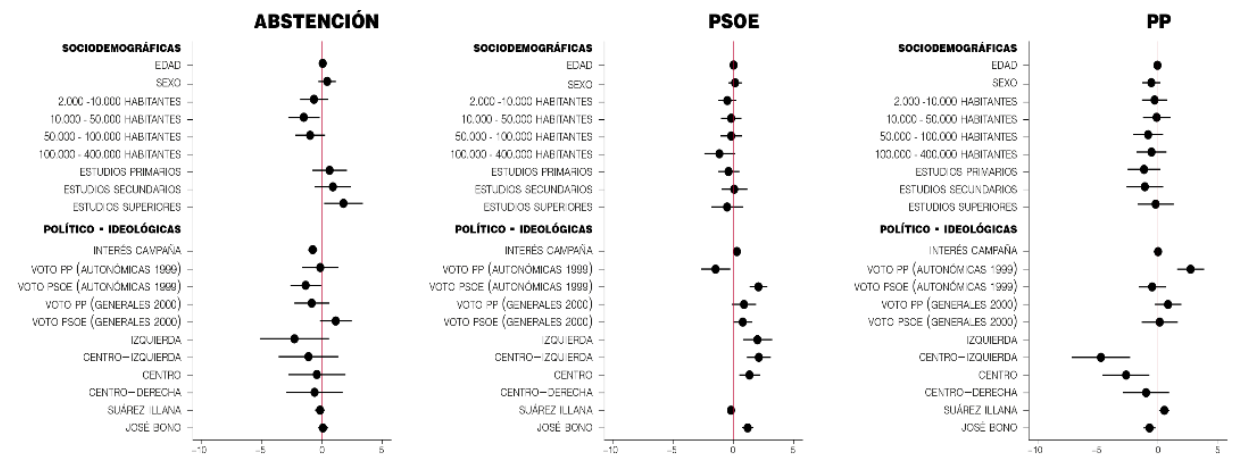

Coeficientes de regresión logística. Elaboración propia. Fuente: Encuesta postelectoral del CIS (n² 2517)

En ese sentido, el modelo explicativo de las elecciones de 2003 muestra un clima de opinión bastante adverso para el Partido Popular (con la posibilidad de un posible voto oculto). En estos comicios en los cuales el Partido Socialista consigue el mayor porcentaje de voto y la mayoría absoluta más fuerte, con José Bono como candidato, tenemos que buscar su hegemonía electoral en, al menos, tres claves: una abstención diferencial, un ensanchamiento ideológico de las bases del PSOE y un liderazgo socialista atrayente por algunos votantes conservadores.

En primer lugar, los estadísticos permiten comprobar el perfil del votante abstencionista: estudios superiores, con poco interés por la información de la campaña electoral, residentes en ciudades pequeñas, pero también, y lo más relevante, votantes del Partido Popular en los pasados comicios autonómicos (1999). Es decir, por un lado, el PSOE consiguió desmovilizar a una parte del electorado de su opositor.

Junto a este hecho, el PSOE consiguió aglutinar una coalición de votantes muy amplio, desde la izquierda hasta el centro (en esta categoría, el 46\% votó al PSOE mientras el 14,1\% lo hizo por el PP); incluso algunos votantes autoubicados como "derecha": el 14,4\% del electorado "centro-derecha" declara haber votado al PSOE, mientras que sólo el $0,8 \%$ del centro-izquierda votó al PP. La explicación, a falta de datos de gestión o de evaluación de liderazgo, debe imputarse a la variable valoración de la campaña de José Bono, con una significatividad superior al 99,9\%. Los votantes socialistas lo valoraron de manera muy fuerte: entre los votantes de estos comicios al PSOE, evalúan su campaña en 4,03 (de 1-5), entre los que votaron PSOE en 1999, un 4,18 y en los que votaron PSOE en las Generales del 2000, un 4,22. También obtiene buenos resultados entre los votantes del PP, especialmente entre aquellos que votaron PP en las Generales del 2000 (José Bono obtiene 3,56, mejor valoración que el candidato popular, 3,29) y en los que votaron PP en las regionales de 1999 (entre este electorado, la campaña de Bono obtiene un 3,29 frente a un 3,36 de la de Adolfo Suárez, prácticamente iguales).

En ese sentido, parece que el candidato popular no fue el más adecuado. En ausencia de más datos y con los resultados que permitan ahondar en más detalles, el PP sólo pudo mantener a una parte de su electorado en 1999, justamente aquellos votantes que evaluaban 
negativamente al candidato socialista (si inferimos los resultados de la variable evaluación de la campaña a una valoración típica de líderes).

Estos patrones se ven reflejados en el modelo estadístico de la segunda convocatoria electoral, la de 2007. El acortamiento en las distancias entre los dos partidos, de veintiún puntos porcentuales en porcentaje de voto a algo más de diez, procede del desgaste del gobierno del PSOE tras más de 24 años de gobierno, un nuevo líder que no consigue atraer lo mismo que su antecesor (José María Barreda) y, sobre todo, a un cambio en los patrones electorales del Partido Popular.

En ese sentido, y referido al primer punto, la victoria del PSOE de Castilla-La Mancha puede asignarse a los buenos datos de gestión. La mayoría de ciudadanos, incluso aquellos votantes del PP, aprueban al gobierno regional: 3,70 (de 1 a 5) de los votantes del PSOE en las Generales del año 2004, frente a 3,04 de los que lo hicieron por el Partido Popular. Sin embargo, empezamos a ver un cierto retroceso en las bases electorales, especialmente en aquellos votantes con mayor nivel educativo, jóvenes y, sobre todo, electores conservadores que en las elecciones generales dan su apoyo al PP pero que confían en los socialistas castellano-manchegos. Por ello, debemos hablar de una cierta pérdida de permeabilidad ideológica de este partido, patrón que sí vimos en 2003, ya que era capaz de atraer votantes declarados como "centro-derecha", pero que ahora desaparece.

Esta polarización política no sólo está referida a partidos, sino también en los liderazgos. El nuevo candidato (José María Barreda), presidente de la Comunidad tras la salida de José Bono (hacia el Ministerio de Defensa), no consigue funcionar de la misma manera que lo hacía su antecesor. Obtiene mejores datos que el incumbent nacional entre los votantes socialistas (su valoración está cerca del 8) y retiene una buena valoración de los conservadores: 5,23 entre los que votaron al PP en las generales en 2004, en cierta medida por el desconocimiento entre sus propios electores de su nueva candidata, María Dolores de Cospedal.

Los datos nos proporcionan una de las principales claves de la campaña: pese a que su nueva candidata ayudó a recortar distancia, todavía era una gran desconocida para la población castellano-manchega: el 25,5\% de la muestra no conocía a la candidata del PP en 2007, entre ellos, el $11,7 \%$ de los que le votaron. Pese a ello, consiguió polarizar los liderazgos y antagonizar con el presidente Barreda, ya que éste ya no obtenía las valoraciones tan positivas de los votantes de PP como las tenía su antecesor, Bono.

Siguiendo con el nuevo liderazgo conservador, María Dolores de Cospedal afianzó a los votantes de derecha y centro derecha en los comicios de 2007, pese a que todavía el PP no fue capaz de ampliar su base ideológica hacia el centro (52,2\% para el PSOE, 20,4\% para el PP). En ese sentido, el Partido Popular fue construyendo varios hitos para que en los siguientes comicios (2011) se alcanzara la victoria: se reforzó en las ciudades medianas (especialmente en aquellas de entre 50.000 a 100.000 habitantes) y atrajo a aquellos votantes de mayor nivel educativo. Sólo fue incapaz de movilizar a una parte de electores que en estos comicios se quedó en casa: aquellos que vivían en ciudades medianas-grandes, con poco interés en campaña, pero que vota en las generales (menor significatividad). 
Gráfico 3 Probabilidad de abstenerse, votar PSOE o PP a las Elecciones de Castilla-La

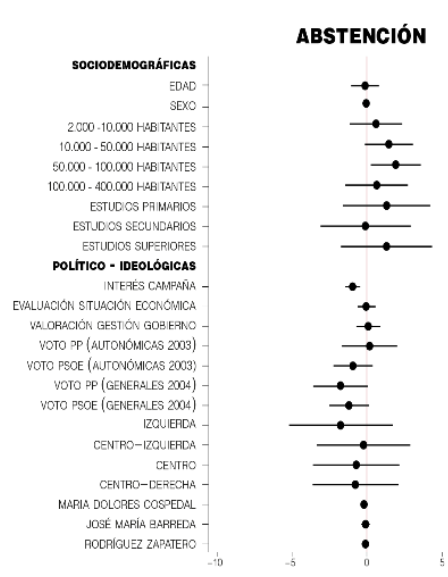
Mancha (2007)

Coeficientes de regresión logística. Elaboración propia. Fuente: Encuesta postelectoral del CIS (n 2712)

\subsection{EL FIN DE LA HEGEMONÍA: EL PRIMER GOBIERNO DE CAMBIO (2011- 2015)}

Con la llegada de la crisis económica a partir de 2008 y la adopción de medidas por parte de los Gobiernos en contra de la opinión pública de ajuste fiscal, numerosos gobiernos europeos sufren duros castigos electorales (Bellucci, Costa Lobo y Lewis-Beck, 2012; LewisBeck y Nadeau, 2012; Magalhaes, 2014). Al igual que en España, donde el voto económico explica en buena medida la derrota a nivel nacional en noviembre de 2011 del Partido Socialista (Martín y Urquizu, 2012; Anduiza et al. 2014), se puede considerar la atribución de responsabilidades al gobierno regional del PSOE con José María Barreda, y con ello, su derrota electoral. La victoria del Partido Popular (explicada no tanto por su incremento electoral, sino por la caída de más de ocho puntos de porcentaje de voto por parte del Partido Socialista), según el modelo de análisis propuesto, se explica por la valoración por parte de sus votantes de su candidata, María Dolores de Cospedal, así como por un ensanchamiento en sus bases electorales. 
Gráfico 4: Probabilidad de abstenerse, votar PSOE o PP a las Elecciones de Castilla-La
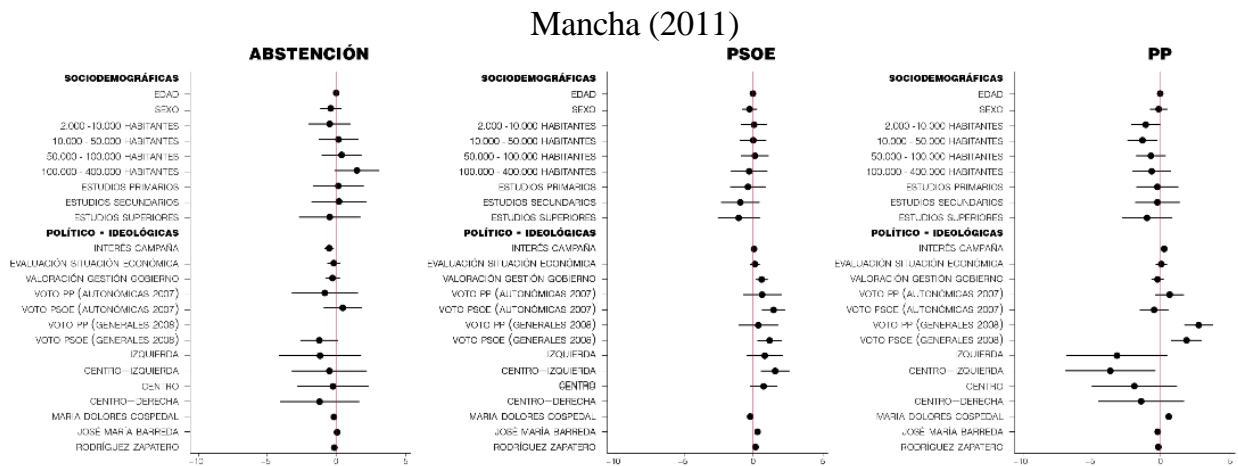

Coeficientes de regresión logística. Elaboración propia. Fuente: Encuesta postelectoral del CIS (n²899)

En primer lugar, observamos una polarización de los liderazgos en ambos electorados. Por un lado, el conocimiento de la candidata de la formación conservadora mejoró en estos años gracias a su proyección nacional (sólo un $0,6 \%$ no conocía entonces a la candidata y Secretaria General del Partido Popular) y con ello su valoración. Como consecuencia, se produjo una mejora en las buenas valoraciones de María Dolores de Cospedal entre todos los grupos de votantes (7,08 en los votantes del PP en las Generales de 2008 y autonómicas de 2007), al contrario que Barreda, que ya no obtenía la simpatía que tuvo cuatro años atrás entre estos votantes $(3,97$ y 3,98 , respectivamente).

En segundo lugar, en el modelo de análisis se observa un ensanchamiento en las bases electorales del PP en tres direcciones: ideológicas, partidistas y territoriales. Por un lado, este partido por primera vez capta más votantes de centro que el PSOE regional (35,1\% por parte del PP, frente al 27,5\% PSOE). Por otro lado, la probabilidad de haber votado a María Dolores de Cospedal entre los votantes del PSOE en los comicios autonómicos de 2007 es favorable significativamente. Y, por último, los datos también certifican una cierta polarización del voto en las zonas rurales-urbanas, siendo estás últimas más proclives a votar al Partido Popular mientras que las más rurales se mantienen afines al Partido Socialista.

Sin embargo, la derrota del PSOE de Castilla-La Mancha por sólo cuatro puntos y un diputado debe ser explicada por una suma de las explicaciones anteriores: su candidato, José María Barreda, era menos permeable en el electorado que hacía un voto diferencial en las elecciones generales (que todavía se mantiene, según el modelo de análisis propuesto) como en las autonómicas anteriores (que ha perdido) por el PP. Esta transversalidad ideológica observada en los modelos de 2003 y 2007 disminuyó moderadamente.

El electorado que mantiene el voto al PSOE regional es aquel con buenas valoraciones tanto del candidato socialista como del incumbent nacional (Rodríguez Zapatero), así como valora mejor la gestión del Ejecutivo regional. Con los resultados que nos ofrecen los estadísticos, ambas explicaciones (la mayor penetración del Partido Popular en los votantes de y en las zonas urbanas, así como la contracción del PSOE en estos dos sectores) permiten explicar el descenso en la valoración de la gestión en los últimos cuatro años, en un momento con una coyuntura económica adversa. 
Gráfico 5: Evolución de la valoración retrospectiva media de la gestión del Ejecutivo regional por ideología y tamaño del hábitat (2007-2011).

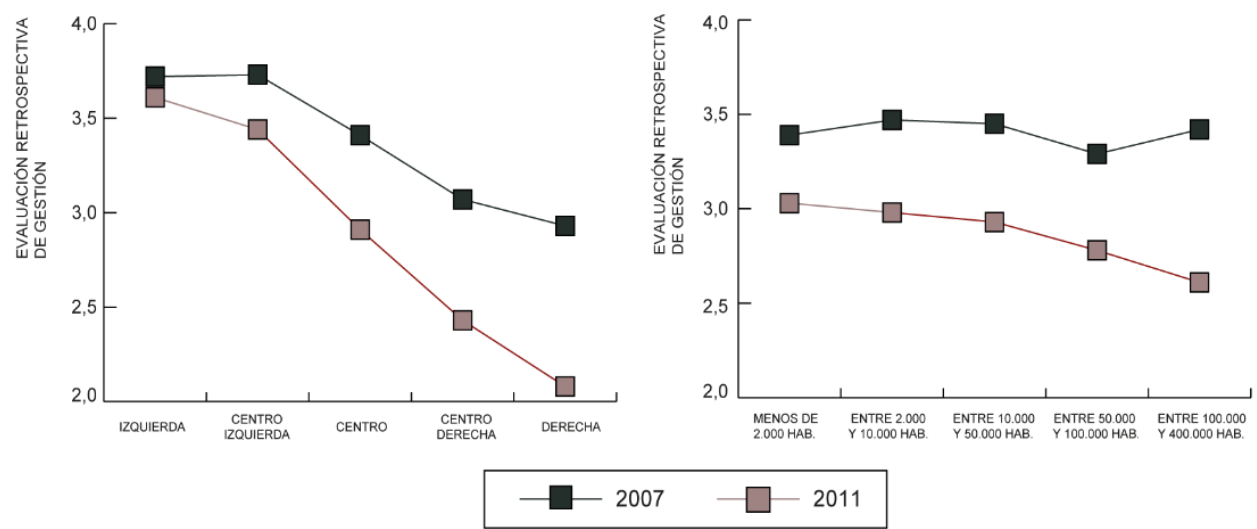

Valoración medida de 1 a 5. Fuente: Encuestas poselectorales del Centro de Investigaciones Sociológicas.

Su valoración media desciende en todas las cohortes de estudio, pero especialmente en dos: por un lado, en los votantes de centro (medio punto) y en el centro derecha ( 0,60 puntos), allí donde el PSOE regional conseguía esa cierta transversalidad ideológica. Pero también en las zonas urbanas (más de 100.000 habitantes), donde su valoración media retrocedió 0,80 puntos frente a 0,40 de media en el resto de zonas. La derrota del PSOE parece responder a la misma pauta por las cuales buena parte de los gobiernos europeos, el nacional y algunos autonómicos, fueron derrotados por la imputación de responsabilidades por parte de sus votantes, que castigaron severamente a los gobernantes por su gestión.

\section{3. ¿LA INSTITUCIONALIZACIÓN DEL PLURALISMO MODERADO (2015- 2019)?}

Con el inicio de la crisis política que rompe el bipartidismo imperfecto a nivel nacional y el inicio de la fragmentación del sistema de partidos tras las Elecciones Europeas de mayo de 2014, los comicios autonómicos anticipan varios meses las tendencias electorales que los sondeos pronosticaban para las Generales de noviembre de ese año. El modelo de análisis para las elecciones regionales en Castilla-La Mancha de mayo de ese año nos ofrece algunas explicaciones para el retroceso de varios partidos, sin perder el foco de que en esta Comunidad la fragmentación partidista fue menor. Podemos sólo consigue dos diputados (Guadalajara y Toledo) y Ciudadanos, pese a superar $12 \%$ en Guadalajara, el 9,5\% en Albacete o 8,45\% en Toledo (donde estuvieron cerca), no consiguieron representación debido a la desproporcionalidad del sistema electoral. Y, si bien la participación electoral sólo cayó en cuatro puntos con respecto a 2011, algunos autores (García, 2013) lo atribuyen a la desafección política de los ciudadanos por la clase política (en línea con los del resto de España), en la medida que ejercieron una mayor abstención como "protesta electoral".

El modelo de análisis muestra la continuidad de algunas de las tendencias que se apuntaron para las elecciones de 2011: polarización del eje izquierda - derecha entre los electorados de 
los dos partidos sistémicos (PSOE y PP), así como buenas valoraciones por parte de sus líderes en sus respectivos electorados (tanto de María Dolores de Cospedal, presidenta desde 2011; como de Emiliano García Page, alcalde de Toledo y candidato por parte del PSOE).

Igualmente observamos que los flujos de voto dual desaparecen en este año: no se observan polos de capilaridad entre ambos electorados, de modo que no existen desalineamientos ideológicos de sus electorados. Los votantes que en las Elecciones Generales apoyaron al PP en 2011 apostaron por Dolores de Cospedal, mientras que aquellos que votaron en las autonómicas y generales por el PSOE en 2011, volvieron a hacerlo en 2015.

Los motivos del retroceso de ambas fuerzas políticas se encuentran en un estrechamiento de sus bases electorales. Con respecto al PSOE, se observa una disminución de los votantes con mayores niveles educativos, siendo su principal electorado los votantes sin estudios; mientras que el PP pierde los electores de las grandes ciudades, en las cuales avanzó significativamente en 2011 y fueron causa de su victoria electoral.

En cambio, la recogida de voto por parte de los dos nuevos partidos parece explicarse más por los rechazos de los ciudadanos a los candidatos de las dos formaciones tradicionales de la región que por motivaciones ideológicas. Las investigaciones de las elecciones de 2015 demostraron que el voto de los nuevos partidos, y, más en concreto en Podemos, estaban conectadas con paliar una serie de necesidades de carácter institucional (representación política, satisfacción con la democracia...). En ausencia de variables concretas de estudio en el cuestionario del CIS y, con las particularidades que ya comentamos en la metodología acerca de la escasa muestra de ambos partidos, parece que el voto a Podemos se correlaciona con la edad (sus votantes son más jóvenes que la media), su adscripción ideológica (habrían votado previamente en las Generales al PSOE y, a nivel autonómico, a IU) y la abstención (en los comicios de 2011). Además, conocen y valoran a su candidato y se posicionan contra el candidato del PSOE, Emiliano García Page.

En cambio, el voto a Ciudadanos se explica por el tipo de hábitat (grandes ciudades), allí donde retrocede el Partido Popular; pero el modelo además muestra correlaciones con haber votado al PSOE y al PP en las autonómicas anteriores y con un rechazo al liderazgo de la candidata popular. 
Gráfico 6: Probabilidad de abstenerse, votar PSOE o PP a las Elecciones de Castilla-La

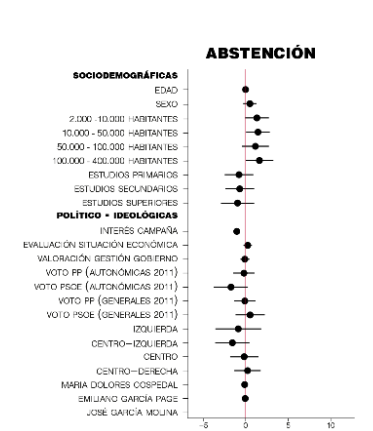
Mancha (2015)
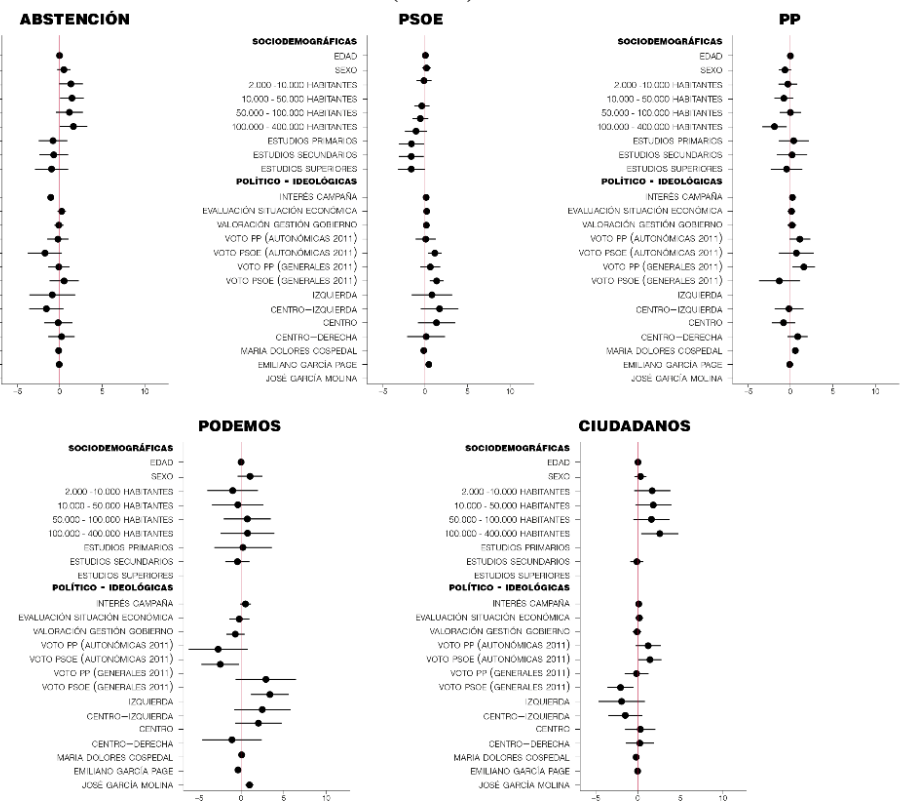

Coeficientes de regresión logística. Elaboración propia. Fuente: Encuesta postelectoral del CIS (nº 3093).

\section{CONCLUSIONES}

El sistema de partidos de Castilla-La Mancha ha sido tradicionalmente estable desde sus inicios en las Elecciones Autonómicas de 1983, debido a aquello que se ha denominado "competición unidimensional por el centro" (Mezo, 2019). Su Estatuto pretendía un modelo bipartidista basándose en un sistema electoral con circunscripciones pequeñas y medianas donde, de facto, se exigía un umbral de representación superior del 8\% que posibilitó que la mayoría de los votos se congregará en dos únicos partidos. Hasta los comicios de 2011, este sistema electoral ha permitido que el PSOE de Castilla-La Mancha gozara de una mayoría absoluta gracias a dos motivaciones: a) una abstención diferencial de los votantes del PP que sí votaban en las Elecciones Generales (planteada en hipótesis $n^{\circ} 1$, y por tanto, verificada); y b) la existencia de un voto dual, diagnosticado y estudiado en Cataluña antes del inicio del proceso independentista (Montero y Font, 1991; Riba, 2000), de un grupo de votantes que apoyaba al PP en las Generales pero que en las Autonómicas lo hacía por el PSOE, especialmente en 2003. Este modelo se debilitó en el año 2007, pero permitió explicar el éxito de los socialistas en la región durante casi tres décadas.

Las explicaciones para ambas tendencias (voto dual y abstención diferencial) parecen extraerse del ensanchamiento de las bases ideológicas del PSOE y, sobre todo, de las valoraciones favorables de su candidato y presidente de la Junta (José Bono, primero, en 2003, y José María Barrera, en 2007) por parte de todo el espectro de votantes. Contrasta que este 
apoyo apareciera incluso en aquellos individuos que apoyaron al PP en las autonómicas (su propio candidato era peor valorado por sus propios votantes, o desconocido, como sucedió en 2007) en la línea con la hipótesis $n^{\circ} 3$, que correlacionaba el éxito del PSOE con la valoración de su candidato por los votantes conservadores.

Sin embargo, la hipótesis relativa al tipo de hábitat $\left(n^{\circ} 2\right)$ parece no convalidarse, al menos con carácter general: este efecto no se confirma en 2003 (el partido estaba ampliamente asentado en todos los tamaños del municipio) y en 2007, la correlación sólo apunta a una ligera reducción en las grandes ciudades. Las elecciones de 2011 confirmaron la dicotomía campo ciudad, siendo el Partido Popular fuerte en las grandes ciudades, mientras el PSOE se mantuvo estable en las localidades más pequeñas y entornos rurales. La gestión de la crisis económica hizo el resto y la valoración del incumbent nacional empujó a una parte de los electores del PSOE a dar un voto de apoyo al Partido Popular. De este modo, se acaba el sistema de partido predominante y el cambio de gobierno se consuma en la región.

Con la reforma del Estatuto en 2014 y la reducción del número de diputados, la Cámara castellano-manchega se anticipó a la crisis política y a la llegada de los dos nuevos partidos. A pesar de ello, el sistema de partidos cambió con la entrada de una tercera formación política, Podemos, que con su apoyo a la investidura de Emiliano García Page no sólo supuso la vuelta del PSOE a la Junta, si no una nueva etapa: hacia un modelo pluralista moderado. Las Elecciones Autonómicas de mayo de 2019 han supuesto un paso atrás. La nueva mayoría absoluta del PSOE puede anticipar una vuelta al sistema de partido predominante (desaparecido ya a nivel autonómico con la fragmentación electoral en todos los niveles) o hacia una estabilización de un sistema bipartidista. En buena medida dependerá de si la reforma del sistema electoral vuelve a la opinión pública. En todo caso, el resultado de estas elecciones es la consecuencia de una polarización partidista que se ha ido fraguando en la serie histórica analizada, con pocas fluctuaciones electorales de votantes entre ambos partidos y que, como colofón, concluye en esta nueva victoria por parte del PSOE.

\section{BIBLIOGRAFÍA}

Álvarez González, J. M. (2003) "Factores de voto en Galicia”. RIPS. Revista de Investigaciones Políticas y Sociológicas, 2(2): 125-148.

Anduiza, E., Bosch, A., Orriols, L., y Rico, G. (2014) Elecciones generales 2011. Madrid: Centro de Investigaciones Sociológicas.

Balcells I Ventura, L. (2007) "Es el voto nacionalista un voto de proximidad o un voto de compensación? una nueva aproximación espacial al voto en dos dimensiones". Revista Española De Ciencia Política, (16): 61-88.

Bellucci, P., Costa Lobo, M., y Lewis-Beck, M. S. (2012) "Economic crisis and elections: The european periphery". Electoral Studies, 31(3): 469-471. DOI: https://doi.org/10.1016/j.electstud.2012.02.009

Cazorla, Á. (2014) Los componentes del voto en andalucía: Un análisis del voto económico en el período 2008-2013. Tesis doctoral. Universidad de Granada.

Castellanos, J.A. (2007) "La transición democrática en Castilla-La Mancha (1976-1983): proceso autonómico y construcción regional”. Toledo: Consejo Económico y Social de Castilla-La Mancha.

Colomer, J. M. (2004) Handbook of electoral system choice. Nueva York: Palgrave Macmillan.

Corujo, A., Fernández-Esquer, C., y Rama, J. (2019) "Quién vota a los partidos nacionalistas en España? Un análisis de las bases electorales de coalición canaria”. Revista Española De Ciencia Política, 51: 39-71. DOI: https://doi.org/10.21308/recp.51.02 
Fernández Esquer, C. (2016) "La reforma del sistema electoral de Castilla-La Mancha de 2014". Cuadernos Manuel Giménez Abad, 11: 76-85.

Gallagher, M. (1991) "Proportionality, disproportionality and electoral systems". Electoral Studies, 10(1): 33-51. DOI: https://doi.org/10.1016/0261-3794(91)90004-c

García, J. (2013) "La participación electoral en Castilla-La Mancha: La abstención y el voto protesta en las elecciones autonómicas (1999-2011)", en Giménez, S. y Tardivo, G.: "Proyectos sociales, creativos y sostenibles". Toledo: Asociación Castellano-Manchega de Sociología (ACMS).

Groba, D. M. y Pereira López, M. (2018) "Los componentes del voto en las elecciones autonómicas de 2015-2016 en Andalucía, Cataluña, Galicia y País Vasco". RIPS: Revista De Investigaciones Políticas Y Sociológicas, 17(1): 55-88. https://doi.org/10.15304/rips.17.1.4973

Lago Peñas, I. (2000) "Identidades duales y abstención diferencial en las comunidades autónomas: los casos de Cataluña, Galicia y País Vasco". Dereito: Revista xuridica da Universidade de Santiago de Compostela, 9(2):83-103.

Lewis-Beck, M. S. y Nadeau, R. (2012) "PIGS or not? economic voting in southern Europe". Electoral Studies, 31(3), 472-477. https://doi.org/10.1016/j.electstud.2012.02.008

Lijphart, A. (1995) Sistemas electorales y sistemas de partidos. un estudio de veintisiete democracias (1945-1990). Madrid: Centro de Estudios Constitucionales.

Llera, F. J. (2006) "Elecciones autonómicas de 2004 en Euskadi: Realineamiento y cambio de ciclo". En Molins, J. y Oñate, P. (Coord.), Elecciones y comportamiento electoral en la España multinivel. Madrid: Centro de Investigaciones Sociológicas, pp. 77-104.

Llera, F. J. (2016) Las elecciones autonómicas en el País Vasco, 1980-2012. Madrid: Centro de Investigaciones Sociológicas.

Llera, F. J., Leonisio, R., y García Ramadán, J. (2008a) "Euskadi 2008: El vuelco socialista". Cuadernos De Alzate: Revista Vasca De La Cultura Y Las Ideas, 38:155-192.

Llera, F. J., Leonisio, R., y García Ramadán, J. (2008b) "El poder territorial y

local en España en 2007", en AA.VV., Sociología y realidad social, Madrid, CIS: 497-563.

Magalhães, P. C. (2014) "Introduction: Financial crisis, austerity, and electoral politics". Journal of Elections, Public Opinion \& Parties, 24(2):125-133. DOI: https://doi.org/10.1080/17457289.2014.887090

Martín Sánchez, M. (2010) "El Estado Autonómico y el procedimiento electoral en España: Reforma de la Ley Electoral de Castilla-La Mancha". Revista de Derecho Electoral, (10).

Martín, I., y Urquizu, I. (2012) "The 2011 General Election in Spain: The collapse of the socialist party”. South European Society and Politics, 17(2): 347-363. DOI: https://doi.org/10.1080/13608746.2012.708983

Mata, T. y Ortega, M. (2013) "Liderazgo y voto: La influencia de los líderes en tres elecciones autonómicas”. Revista Española De Ciencia Política, 31:123-152.

Mena, L. (1998) “Castilla-La Mancha. Elecciones autonómicas”, en Alcántara, M. Y Martínez, A.: "Las elecciones autonómicas en España, 1980-1997”. Madrid: Centro de Investigaciones Sociológicas.

Mezo (2019) "Castilla-La Mancha. Competición unidimensional y bipartidista por el centro, rota por la crisis", en Gómez, B., Cabeza, L. y Alonso, S. (Eds.): "En busca del poder territorial: cuatro décadas de elecciones autonómicas en España". Madrid:Centro de Investigaciones Sociológicas.

Montabes, J. (1996) "La concurrencia electoral en Andalucía". Revista de estudios regionales, 44: 353-370.

Montabes, J. (2015) Análisis del comportamiento electoral de los andaluces en el medio rural y urbano. Actitudes, orientaciones y pautas ante los procesos electorales de 2011-2012. Sevilla: Centro de Estudios Andaluces.

Montabes, J. y Ortega, C. (2008) "Elecciones 2008 en Andalucía: Concentración y continuidad". Colección Actualidad (Centro De Estudios Andaluces), 27: 1-29.

Montero, J. R. y Font, J. (1991) "El voto dual en Cataluña: Lealtad y transferencia de votos en las elecciones autonómicas". Revista de estudios políticos, 73: 7-34. 
Montero, J. R., \& Font, J. (1991) "El voto dual en Cataluña: Lealtad y transferencia de votos en las elecciones autonómicas. Revista De Estudios Políticos, (73), 7-34.

Nohlen, D. (1994) Sistemas electorales y partidos políticos. México: Univ. Nacional Autónoma de México.

Ortega, C., García Hípola, G. y Trujillo, J. M. (2013) "La influencia de la atmósfera política local sobre la conducta electoral. Un estudio del voto socialista en las elecciones locales andaluzas de 2011”. Revista Internacional De Sociología, 71(3): 617-641. https://doi.org/10.3989/ris.2012.04.16

Ortega, C., y Trujillo, J. M. (2015) "Un estudio ecológico del voto socialista en el medio ruralurbano andaluz, 1999-2012”. Revista Española De Investigaciones Sociológicas, 152: 143-166. https://doi.org/10.5477/cis/reis.152.143

Porras, A. (1984) "Geografía electoral de Andalucía". Revista Española De Investigaciones Sociológicas, 28,;151-170.

Riba, C. (2000) "Voto dual y abstención diferencial. Un estudio sobre el comportamiento electoral en Cataluña". Revista Española de Investigaciones Sociológicas, 91: 59-88.

Rico, G. (2002) Candidatos y elecciones: La popularidad de los líderes políticos y su impacto en el comportamiento electoral. Barcelona: Institut de Ciènces Polítiques i Socials.

Rico, G. (2009) Líderes políticos, opinión pública y comportamiento electoral en España. Madrid: Centro de Investigaciones Sociológicas.

Rivera, J. M. y Jaráiz, E. (2016) "Modelos de explicación y componentes del voto en las elecciones autonómicas catalanas de 2015”. Revista Española de Ciencia Política, 42: 13-43. https://doi.org/10.21308/recp.42.01

Ruiz Rodríguez, Leticia M. y Otero Felipe, Patricia (2013) Indicadores de partidos y sistemas de partidos. Madrid: Centro de Investigaciones Sociológicas

Sartori, G. (1976) Parties and party systems: A framework for analysis. Cambridge: Cambridge University Press.

Trujillo, J. M., Ortega, C. Y Montabes, J. (2015) “Tipo de hábitat y comportamiento electoral: Los efectos contextuales sobre la abstención diferencial en Andalucía (2011-2012)”. Revista Española De Ciencia Política, 37: 31-61.

\section{Breve currículo:}

\section{José María Ramírez Dueñas}

Investigador y profesor dentro del Departamento de Sociología Aplicada, especializado en Comunicación Política y Comportamiento Electoral (Universidad Complutense de Madrid). Participa en el grupo de investigación "Comunicación y Política en el Nuevo Espacio Público" (CONEP). Doctor en Sociología por la Universidad Complutense. Sus principales temas de investigación son: análisis de voto en sistemas multinivel, socialdemocracia, efectos de los sondeos en la opinión pública, polarización política y mediática, y la evolución de las campañas electorales.

\section{Agradecimientos}

El autor agradece a los revisores anónimos por los comentarios aportados, ya que han contribuido a mejorar y enriquecer el presente texto. 